\title{
Lombalgie chronique: du diagnostic médical incertain aux étiologies profanes
}

\author{
JOSIANE MBARGA ${ }^{\mathrm{a}}$, CLAUDE PICHONNAZ ${ }^{\mathrm{b}}$, ROSE-ANNA FOLEY ${ }^{\mathrm{c}}$ et CÉLINE ANCEY ${ }^{\mathrm{d}}$ \\ Rev Med Suisse 2018; 14: 850-3
}

\begin{abstract}
Cet article, issu d'une recherche qualitative menée dans un hôpital suisse, est basé sur des entretiens avec 20 participants à un programme de rééducation de la lombalgie. II montre qu'en l'absence de causes objectivables permettant d'expliquer la douleur, les patients élaborent leurs propres interprétations et explications pour donner sens à leur vécu. Leurs modèles explicatifs incluent principalement le mode de vie et les aspects physiques liés au fonctionnement du corps et laissent peu de place à la composante psychologique. Leur interprétation est, par conséquent, en décalage avec la vision médicale actuelle qui considère que les douleurs dans la lombalgie chronique sont d'origine bio-psychosociale. Ce décalage implique une concertation entre patients et professionnels sur les objectifs à poursuivre pour traiter la douleur.
\end{abstract}

\section{Chronic low back pain: from the uncertain medical diagnosis to the profane etiologies}

This qualitative research article is based on interviews with 20 participants to a low back pain rehabilitation program in a Swiss hospital. It shows that, in the absence of the obvious cause that can explain pain, patients construct their own interpretations and explanations in order to give meaning to their experience. Their explanatory models mainly include the lifestyle and the physical aspects related to the body function, what leaves little room for the psychosocial component. Their interpretation is consequently discordant with the current medical approach, which considers that chronic low back pain results from bio-psycho-social factors. This discrepancy implies negotiation between patients and professionals about the objectives to achieve in order to treat pain.

\section{INTRODUCTION}

La prévalence de la lombalgie au cours de la vie est de plus de $80 \%$ dans le monde occidental. Si la douleur se résorbe spontanément chez la plupart des personnes, $23 \%$ d'entre elles évoluent vers la chronicité, et environ 11-12\% sont handicapées. ${ }^{1,2}$ Dans la plupart des situations, les causes spécifiques

aphD Anthropologie médicale / adjointe scientifique HES, Haute école de la santé Vaud (HESAV) / Haute école spécialisée de Suisse occidentale (HES-SO), Unité de recherche en santé, Avenue de Beaumont 21, 1011 Lausanne, bMSc Physiothérapie / professeur associé HES, HESAV/HES-SO, Filière physiothérapie, Avenue de Beaumont 21, 1011 Lausanne, Physiothérapeute clinicien spécialisé, Département de I'Appareil Locomoteur, CHUV-UNIL, 'PhD Anthropologie médicale professeure associée HES, HESAV/HES-SO, Unité de recherche en santé, Avenue de Beaumont 21, 1011 Lausanne, Institut Universitaire de Médecine Sociale et Préventive (IUMSP), Centre Hospitalier Universitaire Vaudois (CHUV), 1012 Lausanne, dMSc Physiothérapie / maître d'enseignement HES, HESAV/HES-SO, Filière physiothérapie, Avenue de Beaumont 21, 1011 Lausanne josiane.mbarga@hesav.ch | claude.pichonnaz@hesav.ch rose-anna.foley@hesav.ch | celine.ancey@hesav.ch de la douleur ne sont pas objectivables alors que les patients attendent un diagnostic clair. ${ }^{1-4}$ «Symptôme sans signe ${ }^{5}$ la lombalgie chronique embarrasse alors autant les patients que les médecins, car tous partagent la difficulté d'objectiver et de catégoriser une douleur au caractère empirique. Face à l'absence d'objectivation des causes de la douleur, les patients construisent des explications et des interprétations pour donner du sens à ce qui leur arrive. Cet article, issu d'une recherche qualitative, s'appuie sur les discours de patients afin de mettre en évidence les étiologies qu'ils attribuent à la lombalgie chronique en l'absence d'explications biomédicales précises sur l'origine des douleurs. Dans une approche biopsychosociale, recommandée pour la prise en charge des lombalgies chroniques, comprendre le point de vue des patients sur les causes de la douleur peut contribuer à mieux prendre en compte leurs représentations, à améliorer l'interaction avec eux et à favoriser l'adhésion aux thérapies.

\section{MÉTHODES}

Les données de cet article proviennent d'une recherche qualitative, menée conjointement par des physiothérapeutes et des anthropologues, auprès de patients souffrant de lombalgie chronique non spécifique (LCNS) et participant à un programme multidisciplinaire de rééducation de trois semaines, dans le Département de l'appareil locomoteur du Centre Hospitalier Universitaire Vaudois (CHUV). Ce programme est proposé à une population active de patients présentant une LCNS, âgées de 18 à 65 ans, sans pathologie psychiatrique sévère ni comorbidités pouvant interférer avec le traitement.

Après l'approbation de la Commission d'éthique cantonale vaudoise, des entretiens semi-directifs ont été menées auprès de vingt patients, sélectionnés selon un échantillonnage raisonné. Cette méthode vise une diversification des profils afin de pouvoir rendre compte de l'influence des caractéristiques du patient sur l'expression de son point de vue. Les guides d'entretien ont été conçus de manière interdisciplinaire, et ont ensuite été affinés à partir de l'analyse de deux entretiens réalisés lors de l'étude préliminaire, d'abord séparément pour chaque profession puis en croisant les points de vue. Les entretiens ont été effectués par les anthropologues. Pour l'analyse des résultats, le logiciel d'analyse de contenu Atlas.ti a été utilisé pour le classement thématique et l'extraction des données, puis l'analyse thématique et l'interprétation ont été réalisées de manière interdisciplinaire, d'abord séparément pour chaque profession, puis en croisant les analyses de manière interdisciplinaire. Les prénoms utilisés dans la suite de l'article sont fictifs. Le tableau 1 présente les caractéristiques de la population étudiée. 


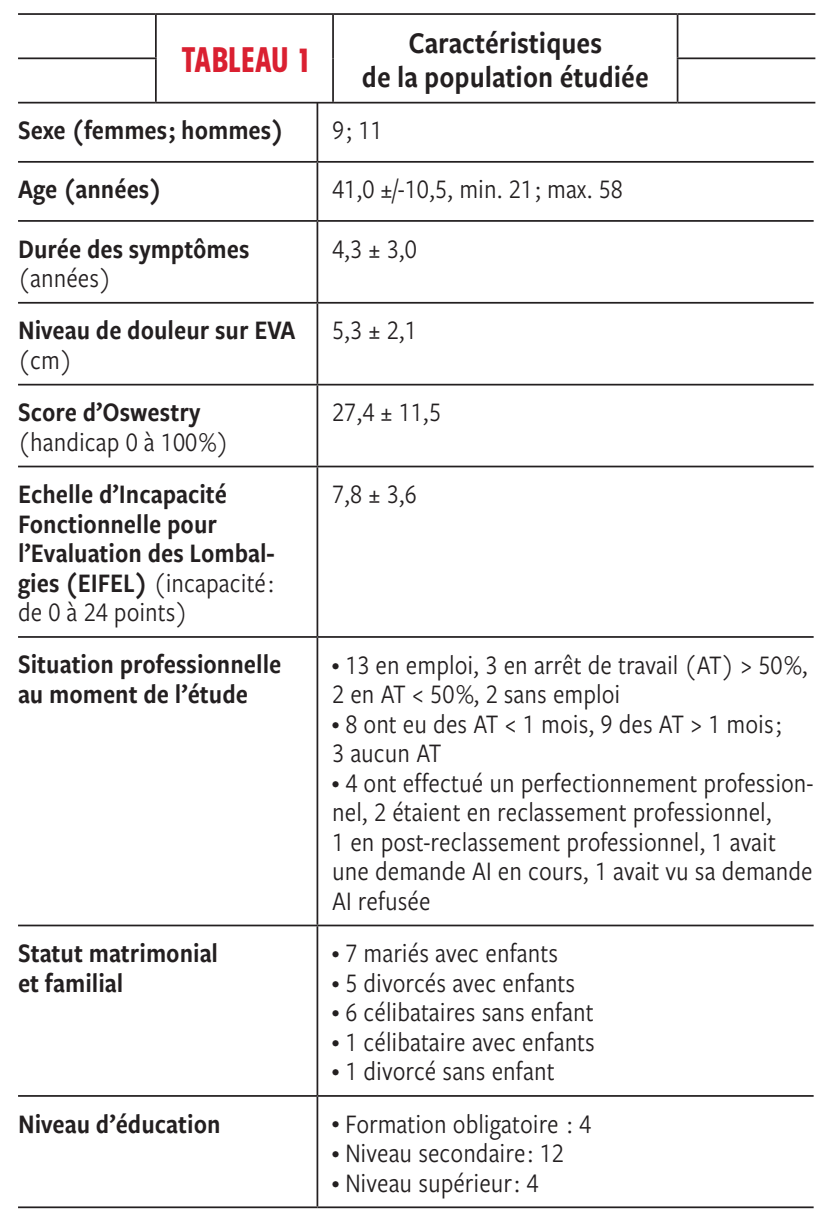

a laissé couler des larmes en consultation. L’incompréhension soulignée par certains patients, comme Luc et Jean (gestionnaire d'équipe), les conduit à consulter différents médecins à la recherche de celui qui les comprendra le mieux.

\section{Les causes de la lombalgie chronique sous le regard des patients}

Face à l'origine incertaine, les participants attribuent des causes variables à la survenue ou à la persistance de la douleur. Intégrant de manière syncrétique les connaissances scientifiques, leurs représentations et leurs vécus, ils construisent un réseau de signification autour de facteurs exogènes ou endogènes, voire des deux. Les facteurs exogènes traduisent les agressions extérieures que subit le corps, tandis que les facteurs endogènes font référence aux résultats de l'imagerie médicale, aux problèmes physiques et aux troubles fonctionnels. Les facteurs évoqués par les patients sont synthétisés dans le tableau 2.

Se situant aux confluents des causes endogènes et exogènes, certains participants évoquent, souvent pêle-mêle ou sous forme de questionnement, une conjonction de facteurs et de causes multifactorielles, sans que leur participation dans la genèse de la lombalgie chronique ne soit claire. Ils mettent l'accent autant sur le travail, les activités physiques exigeantes, le sport, les événements de la vie que sur des problèmes mécaniques et physiologiques, voire génétiques. Les patients expriment parfois des doutes sur la véracité de leur propre regard lorsqu'il ne rejoint pas la vision médicale: «Alors peut-être que c'est moi qui ne vois pas les choses qui ne vont pas (sourire) mais voilà» (Claire, vendeuse).

\section{RÉSULTATS}

\section{Une douleur invisible, des patients qui se sentent incompris}

L'absence d'objectivation et de visibilité de la douleur est source de frustration pour les patients qui éprouvent une certaine délégitimation de leur douleur lorsque les examens ne révèlent pas de cause spécifique. Cette déclaration de Luc, ancien vendeur reconverti en assistant en marketing, illustre l'incompréhension lorsque le ressenti est en décalage avec l'imagerie médicale: «j'avais beau aller voir les médecins en leur disant "J'ai mal au dos", on me disait: "Non vous n'avez rien quoi, on a fait des radios, on a fait des... des choses, y a rien de spécifique, alors oui il y a des étagements, il y a des discopathies, y a un dos qui est très fatigué, qui est très usé, mais bon y a rien de gênant".»

Lorsqu'aucune explication biomédicale ne peut être avancée, la majorité des participants affirment qu'ils se sentent mal compris. Ils évoquent un décalage entre la gravité perçue de leur atteinte et l'attitude de certains médecins qui, disent-ils, à l'instar de Luc, «ne les prennent pas suffisamment au sérieux, considèrent qu'ils font de la comédie ou les qualifient de dépressifs». Cette évocation d'un processus psychique à l'origine des douleurs est parfois réfutée par le patient, et est perçue comme stigmatisante et enfermante. Ainsi, Joëlle, infirmière scolaire, considère avoir été «étiquetée dépressive», suite à un épisode de violentes douleurs au cours duquel elle

\begin{tabular}{|c|c|}
\hline TABLEAU 2 & $\begin{array}{l}\text { Origine des douleurs selon } \\
\text { la perception des patients }\end{array}$ \\
\hline \multicolumn{2}{|l|}{ Facteurs exogènes rapportés } \\
\hline Activité professionnelle & $\begin{array}{l}\text { Métiers à caractère physique: } \\
\text { - Manutention } \\
\text { - Port de charges lourdes } \\
\text { - Longues stations assises ou debout }\end{array}$ \\
\hline $\begin{array}{l}\text { Pratique excessive } \\
\text { du sport dans l'enfance ou } \\
\text { l'adolescence }\end{array}$ & $\begin{array}{l}\text { Sport de compétition ou de haut niveau } \\
\text { - Squash } \\
\text { - Handball } \\
\text { - Vélo } \\
\text { - Moto } \\
\text { - Judo }\end{array}$ \\
\hline $\begin{array}{l}\text { Evénements de vie } \\
\text { difficiles }\end{array}$ & $\begin{array}{l}\text { - Parcours migratoire difficile } \\
\text { - Prise en charge d'un proche malade } \\
\text { - Séparation }\end{array}$ \\
\hline Accident & $\begin{array}{l}\text { - Tomber dans le cadre de son métier } \\
\text { - Tomber dans la rue } \\
\text { - Tomber d'un arbre }\end{array}$ \\
\hline \multicolumn{2}{|l|}{ Facteurs endogènes rapportés } \\
\hline $\begin{array}{l}\text { Atteintes physiques (selon } \\
\text { les mots des patients) }\end{array}$ & 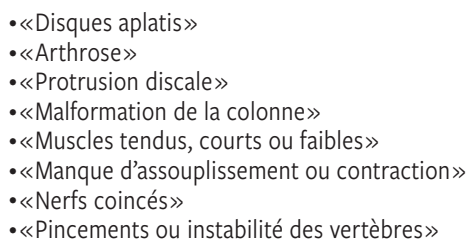 \\
\hline
\end{tabular}




\section{La place perçue des facteurs émotionnels dans l'origine des douleurs}

Si les patients affirment avoir entendu parler du lien entre la douleur et les problèmes psychologiques, la plupart d'entre eux rejette cette éventualité en ce qui les concerne, en affirmant qu'ils ne sont pas centrés sur la douleur, qu'ils n'ont pas eu de surcharge psychologique précédant la lombalgie ou qu'ils ne sont pas de nature stressée. Aucun participant ne considère avoir un profil psychologique qui le prédispose au mal de dos. L'idée d'une faiblesse psychique intrinsèque semble difficilement acceptable pour expliquer leur situation, surtout si elle ne correspond pas à leur perception: «... ce n'était pas une explication quoi, moi je me sentais pas dépressive» affirmait Léa, infirmière anesthésiste. Il arrive que les mêmes personnes reconnaissent que les problèmes émotionnels exacerbent la douleur, sans les considérer pour autant comme des éléments déclencheurs. Ainsi Léa dit: «Oui, moi j’arrive à mettre en lien les situations pénibles psychologiquement où cela se répercute sur mon dos, c'est évident. Si je vis une situation difficile, même maintenant, j'ai encore plus mal au dos quoi». Quelques personnes considèrent les problèmes psychologiques comme étant la résultante plutôt que l'origine des douleurs. Jean affirme «Voilà, quand vous avez mal euh... à raison de 24 heures sur $24,(.$.$) à un moment donné cela rend un peu irritable». Et$ Joëlle ajoute: «De voir quand j'ai mal au dos, à quel point je suis euh... handicapée, entre guillemets, dépendante, c'est ça qui me déprime, et puis la récurrence des crises ça me... ça me déprime». Plus rarement, les problèmes émotionnels et la lombalgie sont vus en interaction réciproque de manière indissociable. Marie, gymnasienne déclare: «Alors les deux sont totalement liés chez moi. C'est clair que si le moral ne va pas bien, le dos ne va pas aller bien non plus, (...) dans l'autre sens aussi, si mon dos ne va pas bien du tout (...) le moral va flancher aussi».

Qu'ils reconnaissent l'interrelation entre la lombalgie et le psychisme ou non, la quasi-totalité des participants expriment la crainte d'être considérés comme des personnes qui ont des problèmes de dos d'origine psychique. Cette catégorisation leur paraît stigmatisante et susceptible d'inciter les médecins à ne plus rechercher les causes physiques de la douleur. Dans cette logique, Joëlle affirme «j'aurais bien aimé qu'elle (comprendre la médecin) aille ... voir pourquoi mon dos il faisait ça, pourquoi ça se bloquait, qu'il n'y avait pas peut-être que le fait que j'étais dépressive.»

\section{DISCUSSION}

Les sentiments de frustration et de délégitimation engendrés par l'absence d'explication physique à l'origine de la douleur ont été rapportés par d'autres auteurs..$^{6-9}$ Cette recherche a de plus investigué les explications profanes que le patient construit pour donner sens à son expérience face à ce vide explicatif.

Les facteurs évoqués, qu'ils soient exogènes ou endogènes convergent vers des explications mécanistes. En accord avec une étude récente, ${ }^{10}$ les patients attribuent généralement l'origine ou la persistance de la douleur à une usure méca- nique liée à la pénibilité du travail, une pratique excessive du sport ou un accident. Leur interprétation est en décalage avec la littérature scientifique qui relève l'importance des facteurs psychologiques. ${ }^{11-14} \mathrm{La}$ composante psychologique est difficile à admettre pour les patients, leurs explications visant fréquemment à pallier l' «accusation» selon laquelle les douleurs seraient «dans la tête». Si l'impact de la douleur sur les émotions est reconnu par certains, la relation inverse est rarement envisagée.

Du point de vue pratique, le refus de certains patients d'entrer en matière sur des origines émotionnelles peut être un obstacle à la prise en charge bio-psychosociale selon les recommandations actuelles. Une discussion faisant émerger leur point de vue sur l'origine de la douleur, en s'intéressant à l'influence qu'ils attribuent aux éléments spécifiques de leur contexte de vie, devrait faire partie intégrante du bilan. Cette étude met en évidence l'importance d'investiguer les liens entre la douleur et la perception des résultats d'examens médicaux, l'adéquation perçue des réponses données, les événements de vie, la situation professionnelle et les périodes de surcharge physique et psychique. Ce bilan des représentations devrait être à la base d'un dialogue personnalisé qui tienne compte des différences de perception entre le patient et les professionnels de santé. Le travail de communication qui s'ensuit est important pour que la prise en charge globale soit acceptée et comprise, et que dans la perception des patients, elle s'attaque effectivement à l'origine de la douleur.

\section{CONCLUSION}

Face à l'incertitude que crée l'absence d'objectivation de l'origine des douleurs, les patients développent des hypothèses pour donner un sens à ce qui leur arrive. Ces explications sont fréquemment en décalage avec les explications bio-psychosociales de la lombalgie chronique qui prévalent dans le monde de la santé. Il est donc nécessaire, pour les professionnels de santé, de saisir les origines attribuées par les patients à la douleur afin de leur permettre de revoir leurs représentations et de comprendre le sens des thérapies proposées.

Cet article a investigué les représentations des patients sur l'origine de la lombalgie chronique avant un programme interdisciplinaire de rééducation. Un autre volet de notre recherche, investiguant les réactions du patient à un programme interdisciplinaire de rééducation, montre que ces représentations peuvent évoluer.

Conflit d'intérêts: Les auteurs n'ont déclaré aucun conflit d'intérêts en relation avec cet article. 


\section{IMPLICATIONS PRATIQUES}

- Les patients, sujets actifs et réflexifs, élaborent des causes de la lombalgie chronique qui leur semblent plausibles, en l'occurrence la situation professionnelle, la pratique excessive du sport, les accidents et les événements de vie difficiles

- Intégrer ces étiologies profanes dans la réflexion du praticien peut permettre aux professionnels de renouveler leur approche du problème, de «soigner» les représentations des patients et d’améliorer l'efficience des thérapies proposées

- En situation de rééducation, une meilleure prise en compte des représentations des patients peut contribuer à diminuer significativement l'anxiété envers certaines activités perçues comme délétères et à renforcer la capacité ainsi que la confiance du patient dans l'exécution des activités

- La qualité de la communication est importante pour aider le patient à saisir, au-delà des facteurs physiques, la place de la composante psychologique dans l'apparition ou la persistance de la douleur

1 Airaksinen O, Brox JL, Cedraschi C et al. European guidelines for the management of chronic non-specific low back pain, COST B13 Working
Group on Guidelines for Chronic Low Back Pain, 2005.

2 Beaudreuil J, Kone H, Lasbleiz S, et al. Efficacité d'un programme de restauration fonctionnelle pour lombalgie chronique: étude prospective sur un an. Rev Rhum 2010;77:291-5.

$3{ }^{* *}$ Genevay S, Balagué F. Diagnostic et pronostic des douleurs lombaires: apport et limites de la clinique. Rev Med Suisse 2017;13:1283-5.

4 **Bouckenaere D. La douleur chronique et la relation médecin-malade. Cah psych clin 2007;1:167-83. 5 **Desfontaines H. Corps souffrant, corps malade. La réception des plaintes lombalgiques en médecine générale. Sci Soc Santé 2012;30:5-22.

6 Glenton C. Chronic back pain sufferers - striving for the sick role. Soc Sci Med 2003;57: 2243-52.

7 *Lillrank A. Back pain and the resolution of diagnostic uncertainty in illness narratives. Soc Sci Med Medicine 2003;57:1045-54

8 Osborn M, Smith JA. Living with a body separate from the self. The experience of the body in chronic benign low back pain: An interpretative phenomenological analysis. Scan J Caring Sci 2006;20:216-22.

9 *Campbell C, Guy A. Why can't they do anything for a simple back problem? A qualitative examination of expecta-

tions for low back pain treatment and outcome. J Health Psy 2007;12:641-52.
10 Roussel NA, Neels H, Kuppens K, et al. History taking by physiotherapists with low back pain patients: Are illness perceptions addressed properly? Disabil Rehabil 2016;38:1268-79.

11 McCarthy CJ, Oldham JA, Sephton R. Expectations and satisfaction of

patients with low back pain attending a multidisciplinary rehabilitation service. Physiother Res Int 2005;10:23-31.

12 Cedraschi C, Allaz AF, Piguet V. Le rôle des représentations de la maladie et de la douleur dans la relation patient-thérapeute. Douleur et analgésie 1998:2:91-5.

13 Crowe M, Whitehead L, Gagan MJ, et al. Listening to the body and talking to myself - the impact of chronic lower back pain: a qualitative study. Int J Nurs Stud 2010;47: 586-92.

14 Bontoux L, Roquelaure Y, Billabert C, et al. Étude du devenir à un an de lombalgiques chroniques inclus dans un programme associant reconditionnement à l'effort et action ergonomique. Recherche de facteurs prédictifs de retour et de maintien au travail. Ann Réadapt Méd Phys 2004;47: 563-72.

* à lire

** à lire absolument 